\title{
A Numerical Approach for Computing Euler Characteristics of Affine Varieties
}

\author{
Xiaxin $\mathrm{Li}^{1}$, Jose Israel Rodriguez ${ }^{2(凶)}\left(\mathbb{D}\right.$, and Botong Wang ${ }^{2}$ \\ 1 University of California, La Jolla, San Diego, CA 92093, USA \\ xil0950ucsd.edu \\ 2 University of Wisconsin-Madison, Madison, WI 53706, USA \\ \{Jose, wang $@ @$ math. wisc. edu
}

\begin{abstract}
We develop a numerical nonlinear algebra approach for computing the Euler characteristic of an affine variety. Our approach is to relate Euler characteristics of a smooth affine variety with the number of critical points using Morse theory. In general, we stratify a variety into the union of smooth affine varieties to obtain results on singular varieties.
\end{abstract}

Keywords: Euler characteristic - Numerical algebraic geometry • Homotopy continuation

\section{Introduction}

The Euler characteristic is one of the most fundamental topological invariants. In the past decade, a series of work appeared which relate Euler characteristics of complex algebraic varieties with the complexity of algebraic optimization problems $[1,5,15,20,21]$. There are several existing approaches to compute the Euler characteristics of complex algebraic varieties [3,6,16,19], each having their own benefits. Our new approach has the following advantages.

1. Our methods directly compute the Euler characteristic of an affine variety without involving any compactification. This is useful because the closure of a smooth affine variety can have singularities along infinity.

2. We stratify and compute the Euler characteristics of smooth affine varieties. In theory, any $d$-dimensional affine variety can be stratified into the union of at most $d+1$ smooth affine varieties. In contrast to the inclusion-exclusion principle, our method does not involve too many varieties.

3. We can tailor the stratification to reduce the degree of each stratum.

A standard method to compute Euler characteristics of complex algebraic varieties is to reduce to the projective hypersurface case. The drawback of this method is that to compute the Euler characteristic of a projective variety, the number of involved hypersurfaces grows exponentially in the codimension.

In contrast, we compute the Euler characteristic of a smooth equidimensional affine variety $X$ by counting the critical points of $\operatorname{dim}(X)+1$ algebraic functions.

BW is supported by NSF Grant DMS-1701305 and the Alfred P. Sloan foundation.

(C) Springer Nature Switzerland AG 2020

A. M. Bigatti et al. (Eds.): ICMS 2020, LNCS 12097, pp. 51-60, 2020.

https://doi.org/10.1007/978-3-030-52200-1_5 
Given a singular complex affine variety, we stratify it into smooth affine varieties to reduce to the smooth case. In theory, we can always stratify a $d$-dimensional affine variety into $d+1$ smooth (possibly not connected) equidimensional affine varieties of dimension $d, d-1, \ldots, 1,0$. So we need to compute the number of critical points of at most $(d+1)(d+2) / 2$ algebraic functions. Our algorithms also have the practical feature of minimizing the degree of the algebraic functions at the expense of increasing the number of functions to consider.

This work is organized follows. In Sect. 2, we recall a theorem to determine the Euler characteristic of a smooth equidimensional variety with a general hyperplane removed by counting critical points of a function. In Sect.3, we provide some key definitions from numerical algebraic geometry. In Sects. $4-5$ we present algorithms for computing Euler characteristics.

\section{Euler Characteristics and Critical Points}

Let $X$ be a topological space that is homotopy equivalent to a finite $\mathrm{CW}$-complex. The Euler characteristic of $X$, denoted by $\chi(X)$, is the alternating sum of the Betti numbers of $X$ [9, Page 146]. We are only interested in the situation where $X$ is a (complex) affine algebraic variety [8, Corollary 6.10]. In this case, the Euler characteristic of $X$ is an alternating sum of cardinalities of several sets of critical points as shown in Theorem 1.

Given a singular affine algebraic variety, it admits a stratification into locally closed smooth subvarieties. We can always refine the stratification into locally closed smooth affine subvarieties. Since the Euler characteristic is additive for a stratification of locally closed subvarieties, it is enough to compute the Euler characteristic of smooth affine varieties. In this paper, we discuss the following two objectives.

1. Compute the Euler characteristics of smooth affine varieties.

2. Find algorithms to stratify singular affine varieties into locally closed smooth subvarieties.

Let $X$ be a smooth subvariety of $\mathbb{C}^{n}$. Let $f: \mathbb{C}^{n} \rightarrow \mathbb{C}$ be a regular function. If $X$ is defined by polynomials $g_{1}, \ldots, g_{l} \in \mathbb{C}\left[x_{1}, \ldots, x_{n}\right]$, then the critical points of $\left.f\right|_{X}$ for a polynomial $f \in \mathbb{C}\left[x_{1}, \ldots, x_{n}\right]$ are the points $P \in X$ such that the vector $\left.\left(\frac{d f}{d x_{1}}, \ldots, \frac{d f}{d x_{n}}\right)\right|_{P}$ is in the linear span of $\left\{\left.\left(\frac{d g_{i}}{d x_{1}}, \ldots, \frac{d g_{i}}{d x_{n}}\right)\right|_{P}: i=1, \ldots, l\right\}$.

This theorem relates the number of critical points to the Euler characteristic.

Theorem 1 ([20]). Let $\ell$ denote a general affine linear function $\ell: \mathbb{C}^{n} \rightarrow \mathbb{C}$ and let $X$ denote a smooth equidimensional affine subvariety of $\mathbb{C}^{n}$. Then

$$
(-1)^{\operatorname{dim}(X)} \chi(X \backslash V(\ell))=\#\left\{\text { critical points of }\left.\ell\right|_{X}\right\}
$$

As a corollary we are able to determine the Euler characteristic of $X$ itself.

Corollary 1. Let $X$ be a smooth subvariety of $\mathbb{C}^{n}$. For $i=1, \ldots, \operatorname{dim}(X)$, let $h_{i}$ denote a general affine linear function $\mathbb{C}^{n} \rightarrow \mathbb{C}$. Then we have the equality

$$
\chi(X)=p+\sum_{i=1}^{\operatorname{dim}(X)}(-1)^{\operatorname{dim}(X)-i+1} \eta_{i}
$$


where $\eta_{i}$ is the number of critical points of $\left.h_{i}\right|_{X \cap V\left(h_{1}, \ldots, h_{i-1}\right)}$ and $p$ is the cardinality of $X \cap V\left(h_{1}, \ldots, h_{\operatorname{dim}(X)}\right)$.

Proof. The additive property of Euler characteristic implies the equality

$$
\chi(X)=\sum_{i=1}^{\operatorname{dim}(X)} \chi\left(X \cap V\left(h_{1}, \ldots h_{i-1}\right) \backslash V\left(h_{i}\right)\right)+\chi\left(X \cap V\left(h_{1}, \ldots, h_{\operatorname{dim}(X)}\right)\right) .
$$

It follows from Bertini's theorem that each intersection $X \cap V\left(h_{1}, \ldots h_{i-1}\right)$ is smooth and $X \cap V\left(h_{1}, \ldots, h_{\operatorname{dim}(X)}\right)$ is a set of $p$ points. By Theorem 1 , we have $\eta_{i}=(-1)^{\operatorname{dim}(X)-i+1} \chi\left(X \cap V\left(h_{1}, \ldots h_{i-1}\right) \backslash V\left(h_{i}\right)\right)$.

\section{Numerical Algebraic Geometry Basics}

In this section we recall a witness set [4,23], which is a fundamental concept in numerical algebraic geometry. A witness set is used to analyze algebraic varieties and is manipulated using homotopy continuation [2], as seen in Sects. 3.2-3.3.

\subsection{Witness Sets and Numerical Irreducible Decomposition}

Let $X$ be an equidimensional subvariety of affine space $\mathbb{C}^{n}$. As a consequence of Bertini's Theorem, there are two invariants, dimension and degree, of $X$ that can be understood by intersecting $X$ with a general linear space. The dimension $\operatorname{dim}(X)$ of a subvariety $X$ of $\mathbb{C}^{n}$ is the codimension of a general affine linear space $\mathcal{L} \subseteq \mathbb{C}^{n}$ such that $X \cap \mathcal{L}$ is finite and nonempty. The degree $\operatorname{deg}(X)$ of $X$ is the number of points in $X \cap \mathcal{L}$.

Definition 1 (Witness set). Suppose $X$ is an equidimensional subvariety of $\mathbb{C}^{n}$. A witness set for $X$ is a triple $(F, L, W)$, where $F$ is a finite set of polynomials with each irreducible component of $X$ being an irreducible component of $V(F), L$ is a set of $\operatorname{dim}(X)$ general $^{1}$ affine linear functions, and $W$ is the set of points $X \cap V(L)$.

In numerical algebraic geometry, $W$ is called a witness point set for $X$. Since $L$ consists of general affine linear functions, the affine linear space $V(L)$ is general and the cardinality of the set $W$ is $\operatorname{deg}(X)$. Throughout, we assume the ideal generated by $F$ in Definition 1 defines a reduced scheme by using deflation $[13,18]$.

Given a (not necessarily equidimensional) subvariety $X$ of $\mathbb{C}^{n}$, we denote by $X_{i}$ the union of $i$-dimensional irreducible components of $X$. We call a set of witness sets of $X_{i}$ for $i=0,1, \ldots, k$ a numerical equidimensional decomposition of $X$. This decomposition can be refined to a numerical irreducible decomposition of $X$ by providing a witness set for each irreducible component of $X$ (see [4]).

\footnotetext{
${ }^{1}$ By "general" here, we mean the intersection $X \cap V(L)$ is transverse and has cardinality $\operatorname{deg}(X)$.
} 
Example 1 (Embedding). Suppose $X$ is an equidimensional subvariety of $\mathbb{C}^{n}$ and $h$ is a linear function $\mathbb{C}^{n} \rightarrow \mathbb{C}$. Let $\widehat{X}$ be the image of $X$ under the closed embed$\operatorname{ding} \mathbb{C}^{n} \rightarrow \mathbb{C}^{n+1}$ given by $x \mapsto(x, h(x))$. Given a witness set $(F, L, W)$ for $X$, we construct a witness set for $\widehat{X}$ as $\left.\left(F \cup\left\{h-x_{n+1}\right\}, L,\{(x, h(x)): x \in W)\right\}\right)$.

Example 2. We can also easily construct a witness set for the Cartesian product of two varieties. Suppose $X_{i}$ is a subvariety of $\mathbb{C}^{n_{i}}$ for $i=1,2$. If $\left(F_{i}, L_{i}, W_{i}\right)$ is a witness set for $X_{i}$, then $\left(F_{1} \cup F_{2}, L_{1} \cup L_{2}, W_{1} \times W_{2}\right)$ is a witness set for $X_{1} \times X_{2} \subset \mathbb{C}^{n_{1}} \times \mathbb{C}^{n_{2}}$.

\subsection{Witness Collections of Subvarieties in $\mathbb{C}^{n} \times \mathbb{C}^{n}$}

A witness collection is a generalization of a witness set and is used to study varieties that are defined by polynomials with a natural multi-variable group structure. For a complete description of witness collections see $[10,11,17]$. For our purposes, it suffices to study the following special case.

A witness collection for a $d$-dimensional irreducible subvariety $Z$ of $\mathbb{C}^{n} \times \mathbb{C}^{n}$ is the following collection of triples, which we call multi-affine witness sets:

$$
\left(F, L^{i} \cup M^{d-i}, Z \cap\left(\mathcal{L}^{i} \times \mathcal{M}^{d-i}\right)\right) \text { for } i=0,1, \ldots, d,
$$

where $F$ is a set of polynomials such that $V(F)$ contains $Z$ as an irreducible component; $\mathcal{L}^{i}$ and $\mathcal{M}^{i}$ are general codimension $i$ affine linear spaces in $\mathbb{C}^{n}$ defined the sets of general linear functions $L^{i}$ and $M^{i}$ respectively.

Witness collections are used to understand the intersection of $Z$ with a Cartesian product of linear spaces. Let $\mathcal{A}^{i}$ and $\mathcal{B}^{i}$ denote general codimension $i$ affine linear spaces in $\mathbb{C}^{n}$. With homotopy continuation, we determine the isolated points in the intersection $Z \cap\left(\mathcal{A}^{i} \times \mathcal{B}^{d-i}\right)$. These points are contained in the set of endpoints of the homotopy $H^{i}: \mathbb{C}^{n} \times \mathbb{C}^{n} \times \mathbb{C} \rightarrow \mathbb{C}^{N+d}$ with

$$
(x, y, t) \mapsto\left(F(x, y), t L^{i}(x)+(1-t) A^{i}(x), t M^{d-i}(y)+(1-t) B^{d-i}(y)\right)
$$

where $L^{i}, A^{i}, M^{d-i}, B^{d-i}$ are sets of affine linear functions defining $\mathcal{L}^{i}, \mathcal{A}^{i}, \mathcal{M}^{d-i}$ and $\mathcal{B}^{d-i}$ respectively. For more details see [10, Remark 1.3]. To conveniently denote linear functions, for $a, x \in \mathbb{C}^{n}$, we take $a \circ x$ to be the usual inner product.

Example 3 (Conormal variety). Let $X=V\left(f_{1}, \ldots, f_{k}\right)$ be a smooth equidimensional variety in $\mathbb{C}^{n}$ with $\left(f_{1}, \ldots, f_{k}\right)$ generating a radical ideal. The (affine) conormal variety of $X$ is a subvariety $\mathcal{C}(X)$ in $\mathbb{C}^{n} \times \mathbb{C}^{n}$ with an ideal

$$
\left\langle f_{1}, \ldots, f_{k}\right\rangle+\left\langle(1+\operatorname{codim}(X)) \text {-minors of } \operatorname{Jac}_{x}\left(x \circ y, f_{1}, \ldots, f_{k}\right)\right\rangle \subset \mathbb{C}[x, y],
$$

where $\operatorname{Jac}_{x}\left(x \circ y, f_{1}, \ldots, f_{k}\right)$ is a $(k+1) \times n$ matrix of partial derivatives with respect to $x$. The dimension of $\mathcal{C}(X)$ is $n$. For a projective formulation, see [22].

A witness collection for the conormal variety of $X$ is given by

$$
\left(F, L^{i} \cup M^{n-i}, W_{i}\right) \text { for } i=0, \ldots, n,
$$


where $V(F)$ contains $\mathcal{C}(X)$ as an irreducible component and

$$
W_{i}:=\mathcal{C}(X) \cap\left(\mathcal{L}^{i} \times \mathcal{M}^{n-i}\right) .
$$

Each multi-affine witness set $\left(F, L^{i} \cup M^{n-i}, W_{i}\right)$ has information about the variety $X$. For example, the dimension of $X$ is the maximal $i$ such that $W_{i} \neq \emptyset$ and the degree of $X$ is the cardinality of $W_{\operatorname{dim}(X)}$. Moreover, for $i=0$, the linear space $V\left(M^{n}\right) \subset \mathbb{C}^{n}$ contains a unique point, say $c$. The set of points $(x, y)$ in $W_{0}$ such that $x \circ c \neq 0$ is the set of critical points of the general linear function $x \circ c$ on $X \backslash V(x \circ c)$. The cardinality of the set $W_{0} \backslash V(x \circ c)$ is the Euler characteristic of $X \backslash V(x \circ c)$ up to a sign. In general, the number of points in $W_{i}$ is an upperbound for the $\eta_{i}$ appearing in Corollary 1.
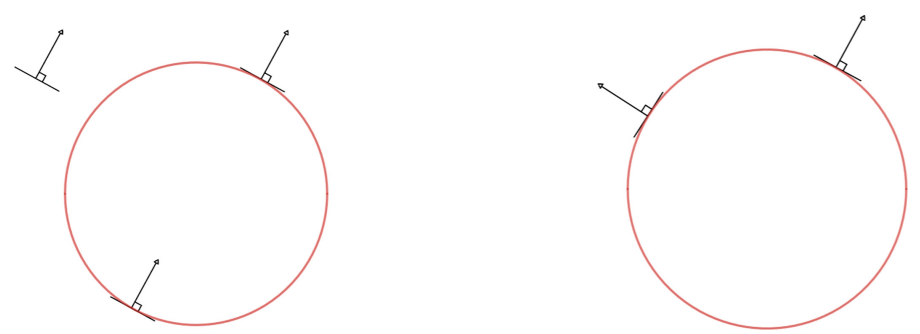

Fig. 1. We illustrate $W_{0}$ (left) and $W_{1}$ (right) for the circle $X=V\left(x_{1}^{2}+x_{2}^{2}-1\right)$ in $\mathbb{C}^{2}$.

\subsection{Regeneration and Removing a Hypersurface}

Given a witness collection for an irreducible variety $X$ and a polynomial $g$, regeneration determines a witness set for $X \cap V(g)$. One of two situations can occur. First, if $X$ is contained in $V(g)$, then $X=X \cap V(g)$ and we are done. Second, if $X$ is not contained in $V(g)$, then for $i=1, \ldots, \operatorname{deg}(g)$ compute a witness set $\left(F \cup\left\{\ell_{i}\right\}, L, W_{i}\right)$ for $X \cap V\left(\ell_{i}\right)$ where $\ell_{i}: \mathbb{C}^{n} \rightarrow \mathbb{C}$ is a general affine linear function. This is easy to do using standard homotopy continuation methods when given the witness set $(F, L \cup\{\ell\}, W)$ for $X$. This produces a witness set $\left(F \cup\left\{\ell_{1} \cdots \ell_{\operatorname{deg}(g)}\right\}, L, \cup_{i=1}^{\operatorname{deg}(g)} W_{i}\right)$ for $X \cap V\left(\ell_{1} \cdots \ell_{\operatorname{deg}(g)}\right)$. Finally, the homotopy $H(x, t)=\left(F(x), t \ell_{1}(x) \cdots \ell_{\operatorname{deg}(g)}(x)+(1-t) g(x), L(x)\right)$ provides a witness set for $X \cap V(g)$ when $t=0$. This procedure for computing a witness set for $X \cap V(g)$ from a witness set for $X$ is called regeneration [11,12,14].

Example 4. For $X \subset \mathbb{C}^{n}$, consider the embedding $\widehat{X} \subset \mathbb{C}^{n+1}$ as in Example 1. Fix a polynomial $g \in \mathbb{C}\left[x_{1}, \ldots, x_{n}\right]$. The affine variety $\widehat{X} \cap V\left(g x_{n+1}-1\right)$ is isomorphic to $X \backslash V(g)$. A useful application of regeneration computes a witness set for $\widehat{X} \cap V\left(g x_{n+1}-1\right)$. From a witness set for $\widehat{X} \subset \mathbb{C}^{n+1}$ and a polynomial $g x_{n+1}-1$, regeneration produces a witness set for $\widehat{X} \cap V\left(g x_{n+1}-1\right)$. 


\section{Euler Characteristics of Smooth Varieties}

Theorem 1 leads to an algorithm that outputs the the Euler characteristic of an algebraic variety by computing critical points. The proof of correctness of the following algorithm is easily derived from Corollary 1.

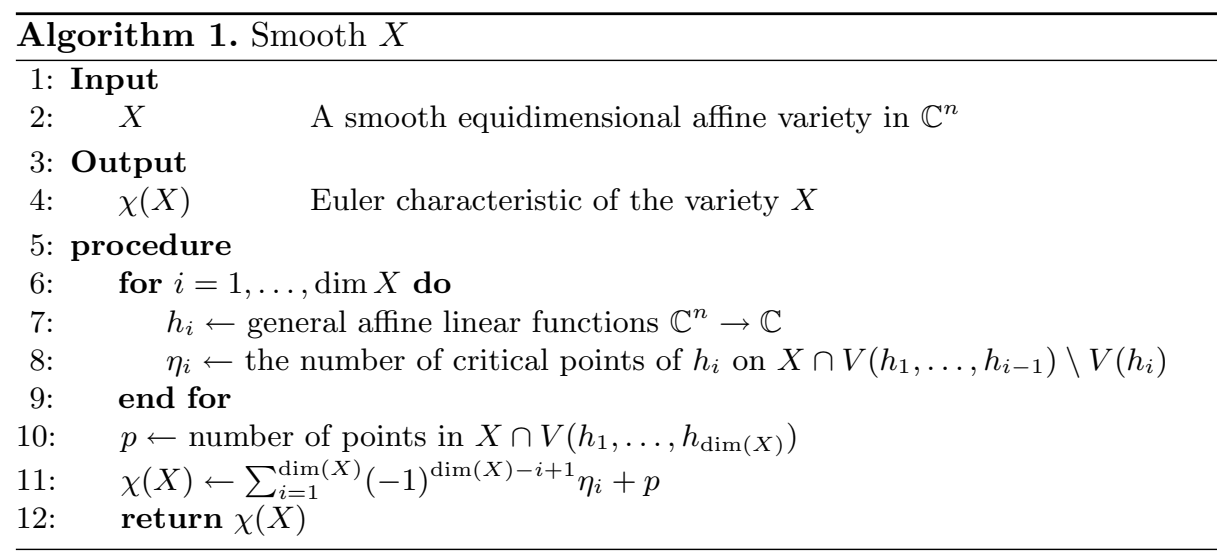

We can compute these critical points from a witness set as follows.

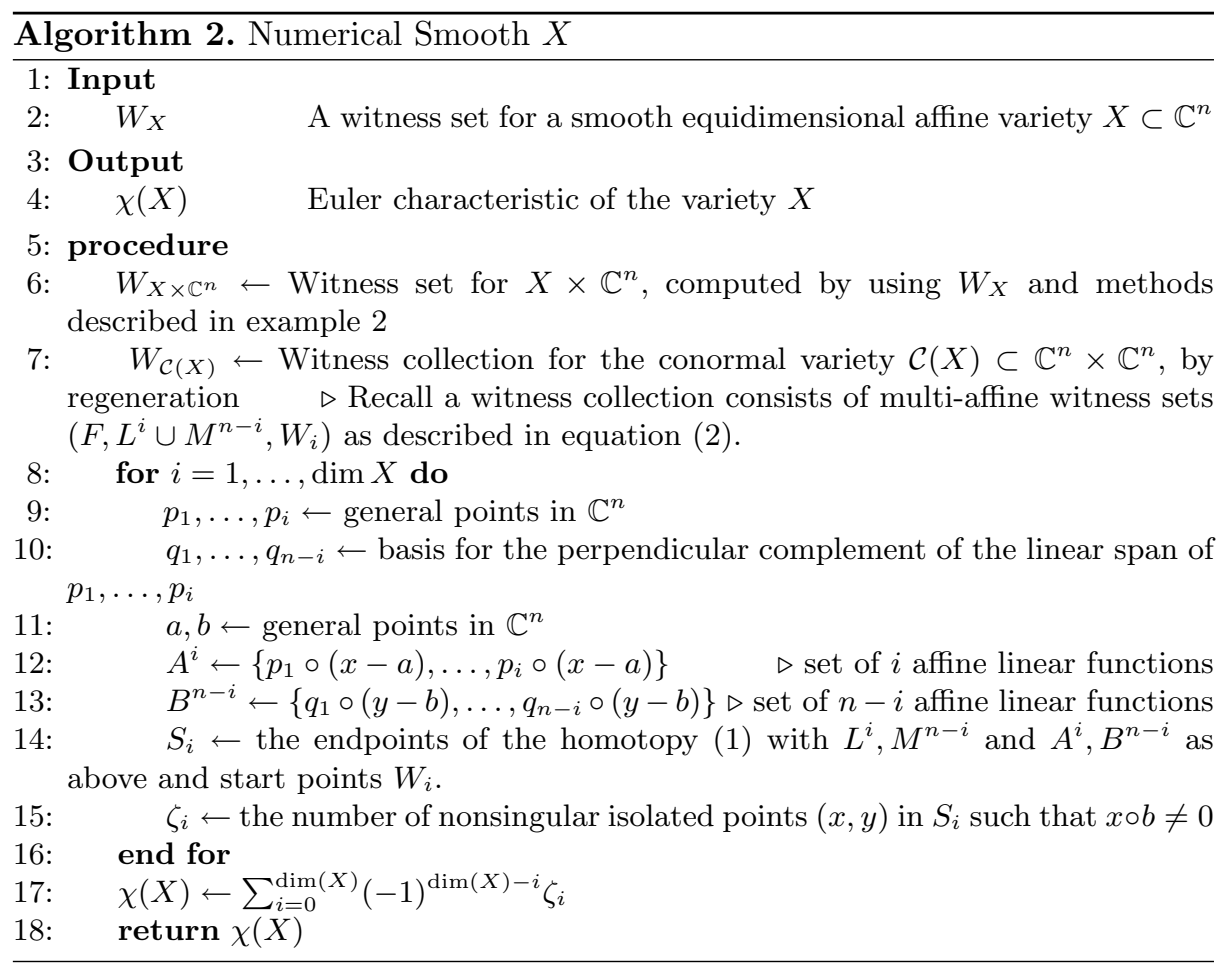


Proof. (Correctness sketch). Example 3 explains how $\zeta_{\operatorname{dim}(X)}=p$ and $\zeta_{0}=$ $\eta_{\operatorname{dim}(X)}$. With some substitutions and algebra one shows $\zeta_{i}=\eta_{\operatorname{dim}(X)-i}$ for $i=1, \ldots, \operatorname{dim}(X)$, and then the result follows.

Example 5. Consider the smooth curve $X=V\left(x_{1}^{2}+x_{2}^{2}-1\right) \subset \mathbb{C}^{2}$ and its conormal variety $\mathcal{C}(X)=V\left(x_{1}^{2}+x_{2}^{2}-1, x_{2} y_{1}-x_{1} y_{2}\right) \subset \mathbb{C}^{2} \times \mathbb{C}^{2}$. The algorithms find $\left(p, \eta_{1}, \eta_{2}\right)=\left(\zeta_{2}, \zeta_{1}, \zeta_{0}\right)=(2,2,0)$ and both output $\chi(X)=0$. The points corresponding to $p$ and $\eta$ are illustrated in Fig. 1 by plotting points on $X$ with the respective normal vectors. The homotopy used to determine $\zeta_{i}$ is given by $H^{i}: \mathbb{C}^{2} \times \mathbb{C}^{2} \times \mathbb{C} \rightarrow \mathbb{C}^{4}$. Concretely, for $H^{1}$, with general affine linear functions $\ell, m: \mathbb{C}^{2} \rightarrow \mathbb{C}$, we have $H^{1}(x, y, t)$ is $\left(x_{1}^{2}+x_{2}^{2}-1, x_{2} y_{1}-x_{1} y_{2},(1-t) \ell(x)+t p_{1} \circ(x-a),(1-t) m(y)+t q_{1} \circ(y-b)\right)$.

\section{Euler Characteristics of Singular Varieties}

In this section, an excision-restriction method to compute $\chi(X)$ is presented.

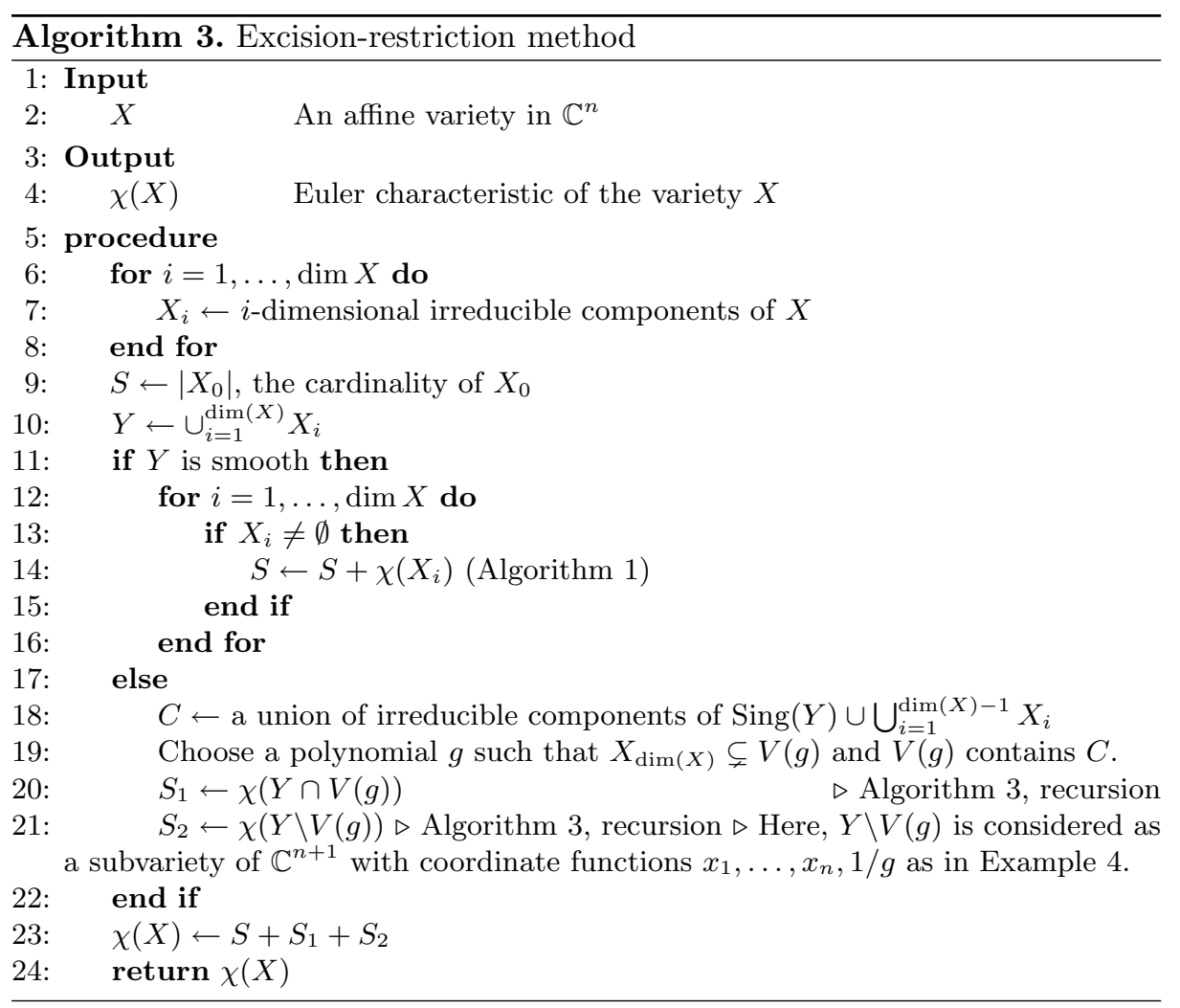

The freedom of choosing $g$ in step 19 is a feature of this algorithm. One way to find such a $g$ is by taking a generic linear combination of minimal generators 
of the ideal of $\operatorname{Sing}(Y) \cup \bigcup_{i=1}^{\operatorname{dim}(X)-1} X_{i}$. An alternative numerical approach is to sample a set of points from an irreducible component of $\operatorname{Sing}(Y) \cup \bigcup_{i=1}^{\operatorname{dim}(X)-1} X_{i}$. to use numerical implicitization, which has been implemented in [7]. To minimize the number of recursions, choose a $g$ vanishing on $\operatorname{Sing}(Y) \cup \bigcup_{i=1}^{\operatorname{dim}(X)-1} X_{i}$. Alternatively, we could choose $g$ so that the degree is small as possible. This heuristic works well for a numerical approach.

Next, we tailor the previous algorithm for regeneration, which was described in Sect.3.3.

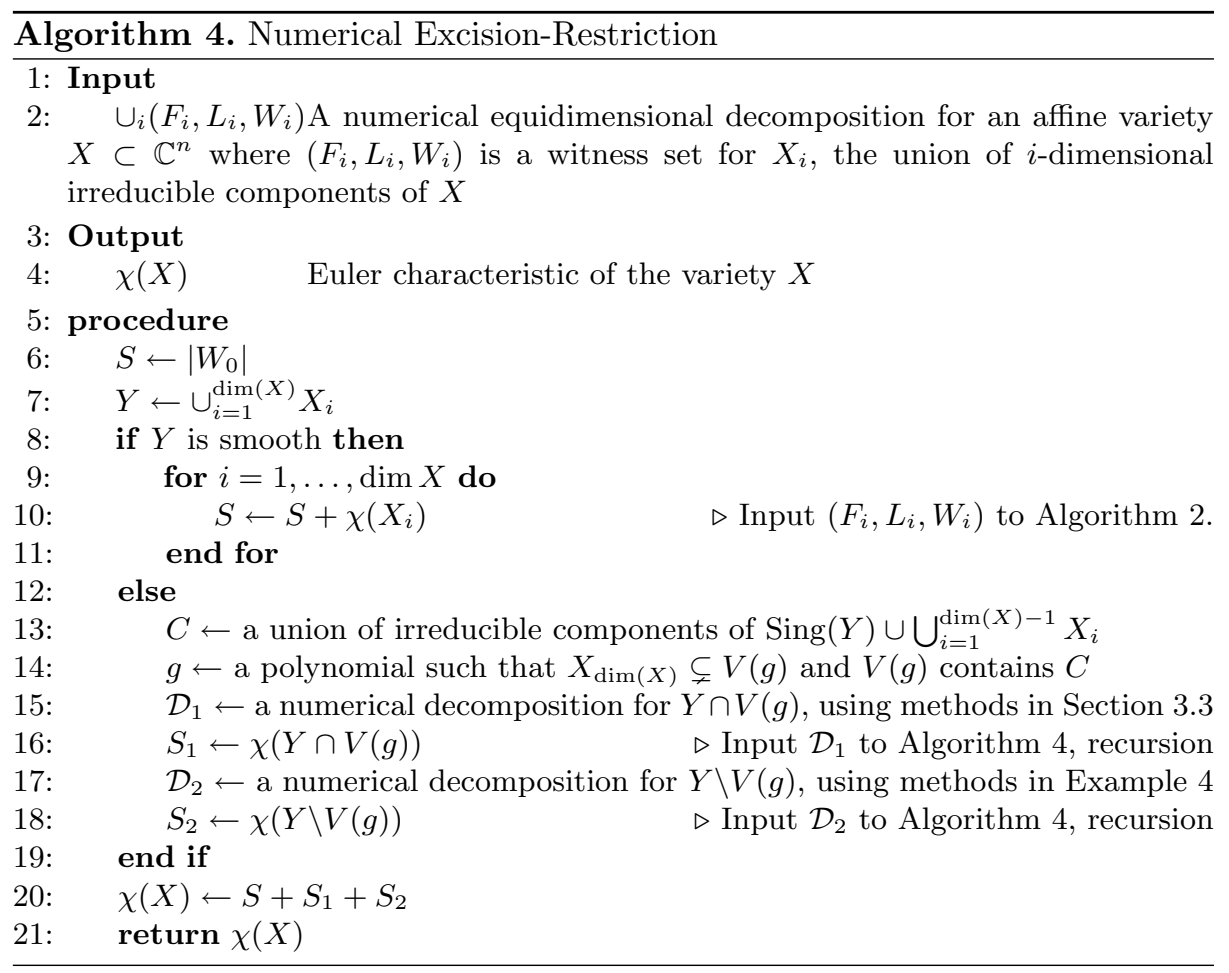

Example 6. Consider the Whitney umbrella $X=V\left(x^{2} y-z^{2}\right) \subset \mathbb{C}^{3}$. The variety $X$ is two dimensional with a singular locus given by the $y$-axis. The output of the algorithm to compute $\chi(X)$ is summarized by the following equations,

$$
\begin{aligned}
\chi(X) & =\chi(X \cap V(g))+\chi(X \backslash V(g)) \\
& =\chi\left(X \cap V(g) \cap V\left(g^{\prime}\right)\right)+\chi\left(X \cap V(g) \backslash V\left(g^{\prime}\right)\right)+\chi(X \backslash V(g)) \\
& =1+0+0,
\end{aligned}
$$

where we have made the following choices: $g=2 x+5 z$ and $g^{\prime}=y-x-4 / 25$ such that $V(g) \supset \operatorname{Sing}(X)$ and $V\left(g^{\prime}\right) \supset \operatorname{Sing}(X \cap V(g))$. 
We input a numerical equidimensional decomposition for $X$. Since $X$ is itself irreducible, we have $Y=X$ and $S=\left|W_{0}\right|=0$. Using regeneration we compute a numerical decomposition $\mathcal{D}_{1}$ for $X \cap V(g)$, which is a union of two lines that intersect at the point $(0,4 / 25,0)$.

In step 16, we apply Algorithm 4 to $\mathcal{D}_{1}$, and in this recursive step we let $g^{\prime}$ play the role of $g$. The variety $X \cap V(g) \cap V\left(g^{\prime}\right)$ is the point $(0,4 / 25,0)$ so we have $\chi\left(X \cap V(g) \cap V\left(g^{\prime}\right)\right)=1$. On the other hand, $X \cap V(g) \backslash V\left(g^{\prime}\right)$ is smooth, so by using Algorithm 2 we get $\chi\left(X \cap V(g) \backslash V\left(g^{\prime}\right)\right)=0$.

Lastly, in step 17, we find a numerical decomposition $\mathcal{D}_{2}$ for $X \backslash V(g)$ to do step 18. Since $X \backslash V(g)$ is smooth, we use Algorithm 2 to find $\chi(X \backslash V(g))=0$.

In this paper we focussed on algorithm development, and we are working towards implementing Algorithm 4. In addition, we are also working to implement Algorithm 3 using Grobner basis to compare with the numerical version and previous techniques.

Acknowledgements. We thank Martin Helmer for very helpful correspondences. We also thank the referees for their helpful comments and suggestions.

\section{References}

1. Adamer, M.F., Helmer, M.: Complexity of model testing for dynamical systems with toric steady states. Adv. Appl. Math. 110, 42-75 (2019)

2. Allgower, E.L., Georg, K.: Introduction to Numerical Continuation Methods. Classics in Applied Mathematics, vol. 25. Society for Industrial and Applied Mathematics (SIAM), Philadelphia (2003)

3. Aluffi, P.: The Chern-Schwartz-MacPherson class of an embeddable scheme. In: Forum Mathematics Sigma, vol. 7, pp. e30 (2019)

4. Bates, D.J., Hauenstein, J.D., Sommese, A.J., Wampler, C.W.: Numerically Solving Polynomial Systems with Bertini. Software, Environments, and Tools, vol. 25. Society for Industrial and Applied Mathematics (SIAM), Philadelphia (2013)

5. Catanese, F., Hoşten, S., Khetan, A., Sturmfels, B.: The maximum likelihood degree. Am. J. Math. 128(3), 671-697 (2006)

6. Chan, C.-Y.J.: A correspondence between Hilbert polynomials and Chern polynomials over projective spaces. Illinois J. Math. 48(2), 451-462 (2004)

7. Chen, J., Kileel, J.: Numerical implicitization. J. Softw. Algebra Geom. 9(1), 55-63 (2019)

8. Dimca, A.: Singularities and Topology of Hypersurfaces. Universitext. Springer, New York (1992). https://doi.org/10.1007/978-1-4612-4404-2

9. Hatcher, A.: Algebraic Topology. Cambridge University Press, Cambridge (2002)

10. Hauenstein, J., Leykin, A., Rodriguez, J.I., Sottile, F.: A numerical toolkit for multiprojective varieties (2019)

11. Hauenstein, J., Rodriguez, J.I.: Multiprojective witness sets and a trace test (2019)

12. Hauenstein, J.D., Sommese, A.J., Wampler, C.W.: Regenerative cascade homotopies for solving polynomial systems. Appl. Math. Comput. 218(4), 1240-1246 (2011)

13. Hauenstein, J.D., Wampler, C.W.: Isosingular sets and deflation. Found. Comput. Math. 13(3), 371-403 (2013) 
14. Hauenstein, J.D., Wampler, C.W.: Unification and extension of intersection algorithms in numerical algebraic geometry. Appl. Math. Comput. 293, 226-243 (2017)

15. Huh, J.: The maximum likelihood degree of a very affine variety. Compos. Math. 149(8), 1245-1266 (2013)

16. Jost, C.: Computing characteristic classes and the topological Euler characteristic of complex projective schemes. J. Softw. Algebra Geom. 7, 31-39 (2015)

17. Leykin, A., Rodriguez, J.I., Sottile, F.: Trace test. Arnold Math. J. 4(1), 113-125 (2018)

18. Leykin, A., Verschelde, J., Zhao, A.: Higher-order deflation for polynomial systems with isolated singular solutions. In: Dickenstein, A., Schreyer, F.O., Sommese, A.J. (eds.) Algorithms in Algebraic Geometry. The IMA Volumes in Mathematics and its Applications, vol. 146, pp. 79-97. Springer, New York (2008). https://doi.org/ 10.1007/978-0-387-75155-9_5

19. Marco-Buzunáriz, M.A.: A polynomial generalization of the Euler characteristic for algebraic sets. J. Singul. 4, 114-130 (2012). With an appendix by J. V. Rennemo

20. Maxim, L.G., Rodriguez, J.I., Wang, B.: Euclidean distance degree of the multiview variety. SIAM J. Appl. Algebra Geom. 4(1), 28-48 (2020)

21. Rodriguez, J.I., Wang, B.: The maximum likelihood degree of mixtures of independence models. SIAM J. Appl. Algebra Geom. 1(1), 484-506 (2017)

22. Rostalski, P., Sturmfels, B.: Dualities. Semidefinite Optimization and Convex Algebraic Geometry. MOS-SIAM Series on Optimization, vol. 13, pp. 203-249. SIAM, Philadelphia (2013)

23. Sommese, A.J., Wampler, C.W.I.: The Numerical Solution of Systems of Polynomials Arising in Engineering and Science. World Scientific Publishing Co. Pte. Ltd., Hackensack (2005) 